\title{
Aktuelle Informationen zur Geographie in der Schule
}

Wir knüpfen hier an die Nr. 1/81 an, wo wir unter diesem Titel Beiträge zusammenstellten, die - wie wir vermuteten - für Lehrer von besonderem Interesse sein dürften.

In den vergangenen Monaten haben wir für diese Rubrik laufend Literatur gesammelt. Einerseits handelt es sich um Publikationen, die ausdrücklich als Unterrichtseinheiten zu einem bestimmten Bereich mit strukturierten Lernzielen verstanden sein wollen, dies v.a. im Zusammenhang mit den in Deutschland seit längerer Zeit laufenen Diskussionen über Curriculum-Inhalte und interdisziplinäre Unterrichtsmethoden.

Andererseits haben wir auch Bücher berücksich- tigt, die sich nicht ausschliesslich an Fachleute wenden, nach unserer Meinung jedoch auch für den Unterricht besonders geeignet sind.

Thematisch können die Werke ganz grob in drei Gruppen eingeteilt werden: die stets wachsende Zahl an Publikationen, die sich mit unserer Umwelt befassen; dann eine Reihe von Neuerscheinungen im geologisch/geomorphologischen Sektor; und eine Anzahl Bücher, die in den Themenkreis "Länderkunde" fallen, was immer man darunter verstehen mag.

Für die Redaktion

Margrit Wegmann

\section{GRUPPE 1: (Unwelt)}

BECK Hartmut/LOB Reinhold E. (Hrsg.) : Umweltschutz im Unterricht. Materialien zur Umwelterziehung, Aulis Verlag Deubner, Köln, 1981.

Lassen wir zu dieser Reihe den Verlag selbst zu Worte kommen: "Umweltschutz im Unterricht" soll eine konkrete Hilfe im Schulalltag bieten. ziel ist nicht wissenshäufung, sondern kritische, fächerübergreifende Auseinandersetzung mit den Problemen unserer gefährdeten Umwelt.

In jedem Heft wird anhand eines konkreten Beispiels ein spezieller Aspekt der Unwelt herausgegriffen und in seiner Problematik abehandelt. Es sind bis jetzt (Stand Mai 1982) folgende Hefte erschienen:

1. Günter Beck: Die Gefährdung stadtnaher Wälder durch Strassenbauprojekte, aufgezeigt am Beispiel Nürnberg.

2. Lothar Geerling/Reinhold E. Lob: Energie und Umwelt am Beispiel der Rheinischen Braunkohle.

3. Helmut Grosu/Manfred Himpsl: Gefährdung einer Wildflusslandschaft durch Energiegewinnung (Beispiel ist hier das Ilztal im Bayrischen Wald) .

4. Gertrud Scherf: Konflike um die Trinkwasserversorgung von Ballungsräumen (Beispiel München).

5. Hanns Lothar Kaempfe/Rudolf Gegelazky: Wie man Energie spart. Es geht hier um Probleme der Isolierung und Heizkostenersparnis.
6. Rosmarie Geiss: Schutz von Feuchgebieten (Beispiel Hochmoor Kendlmühlfilze/Chiemgau).

Die Hefte sind für die Schulstufe des 7.- 10 Jahrgangs gedacht und bringen Stoff aus Biologie, Geographie, Sozialkunde, Geschichte und Physik. Der Vorteil bei einem aktuellen Fallbeispiel liegt zweifellos darin, dass zusätzliches Material aus der Tagespresse, dem Fernsehen oder von Bürgerinitiativen verwendet werden kann, oder dass das Objekt auf einer Exkursion oder gar einer Arbeitswoche besichtigt wird. Es hat für den Lehrer in der Schweiz den Nachteil, dass die Fallstudie ausserhalb unseres Bereichs liegt. Aktualität erhält die Unterrichtseinheit erst, wenn die strukturierten Lernvorschläge mit schweizerischen Daten gefüttert resp. auf ein vergleichbares Objekt in unserem Lande übertragen werden, was einiges an Initiative dem Lehrer abverlangt.

Eigentlich wäre es sinnvoll, wenn die einmal gemachten "Uebertragungen" nicht in den einzelnen Schulen stecken bleiben, sondern auch andern Lehrer zugänglich gemacht werden könnten; dass sich also ein Gremium fände, welches solche "adaptierten" Unterrichtseinheiten veröffentlichen würde.

Der Verlag hat ibrigens noch weitere Hefte in Vorbereitung $\mathrm{zu}$ folgenden Themen: Veränderungen des Klimas durch den Menschen/ Salzbelastung von Werra/Weser und Rhein/ Recycling am Beispiel Papier. 
KYBURZ-GRANER Regula: Schutz des Waldes. Unterrichtseinheit für eine Kooperation der Fächer Biologie/Geographie/Sozialkunde. Institut für Pädagogik der Naturwissenschaften Kiel, Aulis Verlag Deubner, Köln, 1981. Lehrerheft LM 12.80, Schülerheft DM 6.80, Textsammlung DM 9.80.

Sehr begrüssenswert ist die Idee der Autorin, sich fächerübergreifend auf ein aktuelles Thema $\mathrm{zu}$ konzentrieren und eine Unterrichtseinheit zusammenzustellen, die übrigens für Schüler ab 7. Schuljahr gedacht ist.

Im Lehrerheft werden konkrete Vorschläge gegeben wie mit einer Klasse das Thema "Wald" problemorientiert angegangen werden kann; daneben finden sich Rapporte von Schulklassen, die diese Unterrichtseinheit bereits erprobt haben. Das Schülerheft liefert zu den einzelnen Gruppenthemen wie "Geschichte/Forstgesetz" oder "Der Wald als Erholungsraum" strukturierte Arbeitsschritte mit Tabellen zum Selbsteintrag für die Schüler. Die Textsammlung bringt u.a. Auszüge aus dem Deutschen und Schweizerischen Forstgesetz, Zeitungsausschnitte etc.

Als Untersuchungsmethoden werden sowohl solche naturwissenschaftlicher Art (z.B. Messen von Porenvolumen, Nährsalzgehalt des Bodens etc.) vorgestellt, wie auch solche sozialkundlicher Art, wobei ausführlich und allgemein verständlich auf die Umfrage-Methoden mit Fragebogen eingegangen wird.

Da sowohl in den zitierten Texten wie auch in den Beispielen und Zeitungsausschnitten die Schweiz gut vertreten ist, eignet sich diese Unterrichtseinheit auch für unseren OberstufenUnterricht. Als Ergänzung wäre allerdings das Thema des Gebirgswaldes, wie auch der Gebrauch von Luftbildern zur Beurteilung von Waldbeständen und Vegetationskartierungen empfehlenswert. Schwierigkeiten könnten sich ergeben bei der im normalen Unterricht knapp zur Verfügung stehenden Zeit, da oft Arbeitsmethoden - z.B. das Mikroskopieren - vielleicht erst vorgängig erklärt werden müssen. Die Unterrichtseinheit würde sich daher eher für die Durchführung einer Arbeitswoche eignen, an einem Ort, wo auch der Zugang zum Untersuchungsobjekt Wald gewährleistet ist.

CZAYA Eberhard: Ströme der Erde. Aulis Verlag Köln, 1981, 248 S., zahlreiche Figuren, DM 36.--.

Die Themen sind jedem Lehrer geläufig: Karstquellen, Grundwasserspiegel, Thermalquellen, Bifurkationen, Tahlhangbildungen, Urstromtäler, Trichtermündungen, Deltas etc.

Es ist nichts Neues, was Czaya bietet, doch fasziniert mich, eimmal an Beispielen aus verschiedenen Kontinenten ein Thema agehandelt zu sehen, ergänzt durch anschauliche Blockdiagramme oder Kartenskizzen (z.B. die mehrmalige Verlagerung der Hwang-Ho-Mündung), die sich bestens als Vorlage für eine Wandtafelzeichnung oder Hellraumfolie eignen. Im Kapitel "Gebändigte Ströme" beschreibt der Verfasser zahlreiche Arten traditioneller Bewässerung einschliesslich Kanalbauten von der Antike bis heute.

Der Band ist ein Uebersichtsbuch mit guten Illustrationen, leider nicht mehr. Wie man heute ein fast 250-seitiges werk schreiben kann, ohne auf die Problematik der Wasserverschmutzung, der Stauseen, der Trockenlegung von Nassgebieten um nur einige Punkte zu erwähnen - scheint mir etwas wirklichkeitsfremd. Schade, denn eine ebenso differenzierte Handhabung aktueller Bezüge hätte das Buch zu einem umfassenden Nachschlagewerk werden lassen.
LABHART Toni P.: Geologie der Schweiz. Hallwag Taschenbuch Sonderband, 164 S., zahlreiche Abb., Hallwag Verlag Bern, 1982, Fr. 19.80.

Die Foto auf der 1. Umschlagseite hält, was sie verspricht: Ueberblick über den geologischen Aufbau der Schweiz - sachlich, klar verständlich, kompetent.

Es ist erstaunlich, was in diesem kleinformatigen, handlichen Büchlein - das bequem in jedem Rucksack verstaut werden kann - alles Platz findet: von der geologischen Zeittafel auf der innern Umschlagseite, der farbigen geologischtektonischen Uebersichtskarte der Schweiz, über Jura-Profile, Deckenquerschnitt-Profile bis zu einem Uebersichtskärtchen von "Stärke und Verbreitung der alpinen Metamorphose", um nur eini- ge grafische Darstellungen herauszugreifen. Der Verfasser hält sich an die klassische Dreiteilung Mittelland - Jura - Alpen, doch versteht er es ausgezeichnet, in den entsprechenden Kapiteln das Verständnis für den Ablauf sowohl der tektonischen Bewegungen wie auch der mannigfaltigen Erosionstätigkeit zu wecken. Flugbilder und dazugehörige Skizzen neben ausgezeichneten Fotos der Gesteinsarten illustrieren auf beste Weise den Text, wobei lobenswerterweise die Abbildungen der Gesteine gleich beim jeweiligen Abschnitt im Text zu finden sind.

Sehr interessant - weil meist in anderen Publikationen nicht zu finden - sind die Abschnitte "Rohstoffe" (z.B. mit Förderungszahlen während des 2. Weltkrieges und heute), "Meteoritenfälle", "Geologische Karten" und "Zerrkluftmineralien". Als ebenfalls nicht übliche Abbildungen stechen 
die Nr. 4: Schichtfolgen von fünf Erdöltiefbohrungen im Schweizerischen Mittelland, Nr. 15: Ausschnitt aus der Hydrogeologischen Karte der Schweiz wie auch Abb. 24: zyklische, durch wiederholte untermeerische Rutschungen entstandene Schichtfolge im Flysch, ins Auge. Labhart hat ausserordentlich gekonnt den Stoff vereinfacht und in allgemein verständlicher Sprache geschrieben, ohne die Komplexität zu vernachlässigen. Er hat eine Zusammenfassung der Geologie der Schweiz geschaffen, die nicht auf differenzierende Feinheiten verzichtet.

Dabei ist auch die Aktualität des Büchleins hervorzuheben: neueste Forschungsergebnisse sind integriert, Bestimmungsmethoden mit Zahlenmaterial belegt. Andererseits geht ein Eingangskapitel auf die Pioniere und die mühsame Entwicklung der geologischen Forschung ein.

Das Buch scheint mir sowohl für den Laien, der auf Wanderungen mehr Aufschluss über den Aufbau der Schweiz erhalten möchte, wie als "Gedächtnisstütze" für Mittelschüler und Studenten gleichermassen geeignet. Es schliesst eine Lücke, die über Jahre hinweg bestand: ein leichtfassliches Büchlein über den komplexen Stoff, und zusätzlich auf eine Weise verfasst, die alle Erwartungen übertrifft!

HEIERLI Hans: Die Alpen. Kosmos Wanderführer, 424 S., 481 Farbfotos, 111 Kartenskizzen, Ott Verlag Thun, 1982, Fr. 34.--.

Rechtzeitig auf die Bergwanderzeit ist dieser Wanderführer durch die Alpen erschienen. Eine Uebersichtskarte zeigt die vom Verfasser vorgenommene Einteilung des Gebirgsbogens in $12 \mathrm{Re}-$ gionen: Südliche französisch-italienische Westalpen; Nördliche französisch-italienische Westalpen; Westliche Schweizer Alpen; Zentralschweiz und Tessin; Ostschweizer Alpen, Graubünden und Liechtenstein; Allgäuer Alpen und Vorarlberg; Tirol und Osttirol; Salzburger Alpen und Salzkammergut; Kärnten und Jugoslawische Alpen; Oestliche Alpen; Bayerische Alpen.

Heierli nennt für das Jahr 1978 folgende Zahlen: 40 Mio Feriengäste +60 Mio Wochengäste, die im Alpenraum Uebernachtungen buchten (1938 waren es 10 resp. $13 \mathrm{Mio}$ ) 1976 bestanden in der Schweiz und in Desterreich rund 5500 Bergbahnen und Skilifte (450 im Jahr 1938). Dieser Entwicklung, die eine fortschreitende und nicht wieder gutzumachende Zerstörung einer einmaligen Naturlandschaft zur Folge hat, möchte der Autor mit seinem Buch entgegenwirken. Er hat bewusst Wanderungen ausgewählt, die von jedermann - einschliesslich Kindern - unternommen werden können. Auch die Hinweise auf Pflanzen-, Tier- und Mineralienvorkommen sollen Anregungen sein, sich im einzelnen Fall in ein Bestimmungsbuch zu vertiefen. Damit möchte Heierli dazu beitragen, dass einer immer grösser werdenden $\mathrm{Zahl}$ von Wanderern die Schönheit, daber auch die Verletzlichkeit der Natur im Alpenraum bewusst wird.
Auf knapp 20 Seiten gelingt es dem Geologen im Kapitel "Die Alpen aus geologischer Sicht" sein Fachgebiet kurz und prägnant darzulegen. Farbige Skizzen und Querprofile wie auch thematisch ausgezeichnet ausgewählte Farbfotos tragen zur allgemeinen Verständlichkeit bei.

Je ein Einführungskapitel ist den Mineralien, der Gestaltung der alpinen Oberfläche, dem Klima der Pflanzen- und der Tierwelt gewidmet; wiederum sehr gut illustriert mit entsprechenden Farbfotos, die übrigens im Kapitel "Mineralien der Alpen" so hervorragend sind, dass sicher nicht wenige Leser $\mathrm{zu}$ begeisterten Mineraliensammlern werden.

Schlussendlich werden die eigentlichen wanderungen vorgestellt: eine geographische Uebersichtsskizze der jeweiligen Region mit Besonderheiten hinsichtlich Klima, Pflanzen, Tiere und bestehende Nationalpärke. Zu jeder Wanderung folgt eine weitere Skizze (die allerdings eine genaue Karte nicht ersetzen kann!) und Angaben iber Ausgangsund Endpunkt der Wanderung, Marschzeit, Verpflegungsmöglichkeiten sowie ein knapper Marschbeschrieb. Alles übersteigt nie den Umfang einer Seite, so dass bequem eine Fotokopie auf die Exkursion mitgenommen werden kann.

Interessierte finden im Anhang einen Abschnitt "Erklärung geologischer Fachausdrücke", ein Sachregister, ein Ortsregister und ein Literaturverzeichnis.

Der Bilderreichtum von bizarren Felsformationen, vegetationsreichen Talsohlen, atemberaubenden Ausblicken, von Fossilien, Mineralien usw. vermag sicher jeden zu faszinieren, für den fachlich Interessierten ist er jedoch bestens ausgewählte Illustration. Der bescheidene Preis des Buches unterstützt die Absicht des Autors, möglichst viele zum Wandern auch in abgelegenere Gebiete der Alpen zu verleiten.

BINZEGGER R./GRÜTTER E.: Die Schweiz aus dem All. Geographica Bernensia S 7, Geogr. Institut der Universität Bern, 1981, Fr. 8.--.

Wir haben diese Broschüre in diese Gruppe eingereiht, da sie vor allem das Erkennen von tektonischen und geomorphologischen Einheiten anhand von Satellitenbildern zur Sprache bringt. Ein Einführungskapitel orientiert über die Entstehung von Satellitenbilder, es folgen 2 Fotopaare "Trub" udn "Uebeschi", ein Uebersichts-Satellitenbild der Schweiz mit Folie der tektonischen Einheiten. Weiter eine Geologische Karte und 3 Querprofile in Farbe und ein Kapitel über Tektonische Strukturen im Jura.

Für den Unterricht wären weitere Verwendungsmöglichkeiten z.B. für Siedlung, Verkehr sinnvoll gewesen. 
ZBÄREN Ernst: Bergseen der Alpen. Ott Verlag Thun, 142 S., 95 Fotos, 1981, Fr. 35.--.

Der Autor dieses Buches ist weder Geograph noch Biologe, er ist engagierter "Laie", wenn man in diesem Fall das wort noch gebrauchen darf. Die faszinierenden Fotos von Alpenseen sind seine Auswahl aus wohl Tausenden von Aufnahmen, die er alle selbst gemacht hat, und die für ihn die Erinnerung an einen ganz bestinmten Tag, ja eine ganz bestimmte Stunde bedeuten. Sie spannen den Bogen der Seenwelt von den Berchtesgadener Alpen über Hohe Tauern, Rofan, Karwendel, die Detztaler und Zillertaler Alpen, die Ortlergruppe und die Dolomiten, über Bündner und Allgäuer Alpen, Gotthard und Berner Alpen bis ins Wallis und Chablais.

Für jeden Abschnitt des Buches wurden bewusst eher unbekanntere Seen ausgewählt und auf einer Uebersichtskarte verzeichnet.

$\mathrm{Zu}$ jeder vorgeschlagenen Seenwanderung gibt der Autor einen ausführlichen Tourenbeschrieb mit Zeittabelle und Vorschlägen zum Kartenmaterial. Es fliessen aber auch ganz persönliche Erlebnisse ein, sei es die Enttäuschung, einen See nach muihsamem Aufstieg noch unter einer Schneedecke zu finden, sei es ein unvermutetes Auftauchen einer Kreuzotter oberhalb des Morteratsch gletschers oder ein Wettlauf mit Gewitterwolken zum Spinnsee, welche übrigens gewannen!

Im Anhang findet sich noch ein Kapitel über "Leben an und in Bergseen".

\section{GRUPPE 3: (Länderkundliche Themen)}

ECKART Karl: Landwirtschaftliche Probleme europäischer Länder. Materialien zur Geographie, Verlag Diesterweg/Sauerländer, 112 S., 1981, Fr. 11.80 .

Der Autor hat dieses handliche Büchlein verfasst, um - wie er in der Einleitung schreibt - dem Lehrer Material für bestimte Lernziele der Oberstufe im Fach Geographie (nämlich "Raumstrukturen und ihre Veränderung unter dem Einfluss wirtschaftlichen Handelns, bevölkerungsgeographischen Wandels und politischen Handels") zur Verfügung zu stellen. Die Uebersicht ist in vier Hauptabschnitte gegliedert: Landwirtschaft in der $B R D$, in $\operatorname{der} B G$, in der DDR und in den Ländern Ostmitteleuropas.

Im Kapitel der BRD werden vor allem die auch für die Schweiz aktuellen Begriffe wie Sozialbrache, Grenzstandorte, Agrarprograme des Bundes, Flurbereinigung mit Aussiedlungen und Aufstockungen erklärt und zum Schluss die verschiedenen Arten von landwirtschaftlichen Zusammenschlüssen (Genossenschaften, Maschinenringe und -gemeinschaften sowie die beim heutigen Trend zur Kleinfamilie auch im bäuerlichen Betrieb nützliche Einrichtung der Betriebs- und Familienhilfe) vorgestellt.
Ein liebenswertes Buch, das sicher in dem Leser den wunsch weckt, öfter an klaren wassern auf luftiger Höhe zu verschnaufen. Für den Unterricht bietet sich eine Fülle von Ideen, wohin eine Exkursion oder Schulreise gehen könnte.

KARSCH Karl/MUNTWILER Ewald: Der Schweizer Jura und seine Fossilien. 136 S., 127 Abb., Ott Verlag Thun, 1981, Fr. 39.50.

Untertitel: Ein Wegweiser für den Liebhaber. Gute Abbildungen von Fossilien, jedoch Fotos zu kleinräumig, Flugaufnahmen wären besser. Es fehlen genaue Bestimmungsmerkmale der Fossilien, erwïnscht wären auch Angaben zur Lebensweise. Fachausdrücke und Stichwörter sind nirgends zusammengefasst und erklärt. Auch praktische Ratschläge für den Anfänger als Fossiliensammler wären sinnvoller gewesen als moralische Ermahnungen.

PAPE Hansgeorg: Leitfaden zur Gesteinsbestimmung. 152 S., 65 Abb., 9 Tab., Verlag Ferdinand Enke Stuttgart, 1981, DM 12.80.

Eine verwirrende Zusammenstellung von Gesteinsbestimmungs-Methoden, Lupenvergrösserungen von Dünnschliffen, Skizzen und Zeichnungen; keine Fotos! Man fragt sich, wer da das Zielpublikum sein soll?

Das zweite Kapitel ist den ZieJ.en der EG und vor allem den gegenwärtigen Problemen (z.B. der Ueberproduktion) gewidmet, mit besonderer Berücksichtigung der Problematik bei Neueintritten und der "AKP-Staaten".

Im nächsten Kapitel wird den verschiedenen Etappen der Umgestaltung der Landwirtschaft der DDR seit 1945 Rechnung getragen und über Lebens- und Arbeitsbedingungen in der Landwirtschaft heute in diesem Staat berichtet.

Das vierte Kapitel schliesslich befasst sich mit der Entwicklung der Landwirtschaft in vier ausgewählten Oststaaten (Polen, Tschechoslowakei, Ungarn, Rumänien) und ihren Problemen. $\mathrm{Zu}$ jedem Kapitel werden Zahlen geliefert, Fallbeispiele genannt und ein Fragenkatalog für die Schüler aufgestellt.

GRUPP Monika: Fremdenverkehr im Mittelmeerraum. Schulgeographie in der Praxis, 132 S., 15 Abb., Aulis Verlag Köln, 1980, DM 24.--.

Die Autorin hat das Thema "Fremdenverkehr" als Bestandteil eines neuen Erdkunde-Curriculums gewählt, da es den meisten Schülern vertraut ist. Der Mittelmeerraum als Region wurde dabei als "klassisches" Fremdenverkehrsgebiet ausgesucht, und die spanische Ostküste als Ziel vie- 
ler Ferienreisenden aus Deutschland. Die Unterrichtseinheit ist für das 7. Schuljahr und für 8 Lektionen konzipiert.

Im Rahmen der deutschen Curriculum-Diskussion hat sich die Verfasserin auf folgende Zielsetzungen festgelegt:

\section{a) Kognitive Lernziele}

Hier möchte sie vor allem erreichen, dass die Schüler Interdependenzen zwischen Reisemotiven, Zielgebieten und Organisationsarten einer Reise erkennen, dann Kenntnisse über naturgeographische Voraussetzungen und ökonomische Gründe für die Erschliessung des Mittelmeerraums als Fremdenverkehrsgebiet erhalten, und sich zudem der landschaftsverändernden, ökonomischen und sozialen Auswirkungen des Tourismus bewusst werden.

\section{b) Instrumentelle Lernziele}

Diese werden - wie die Autorin erwähnt - im Geographie-Unterricht meist vernachlässigt. Sie versteht darunter, dass die Schüler mit Säulen-, Kurven- und Kreisdiagrammen, mit Statistiken, Fragebogen, Karten, Bildmaterial, Zeitungsartikeln und Fernsehfilmsequenzen umgehen lernen.

\section{c) Affektive Lernziele}

Hierzu gibt die Verfasserin zu, dass sich die geographische Fachdidaktik bisher nicht mit solchen Lernzielen befasst hat. Sie interpretiert sie folgendermassen: die Schüler sollen eine differenziertere Haltung gegenüber ihren eigenen Lebens- und Freizeitbedingungen einnehmen, sie sollen ihre bisher unreflektierte Meinung gegenüber dem Mittelmeerraum überprüfen und eine kritische Haltung gegenüber den Versprechungen der Fremdenverkehrswerbung zeigen können.

Im zweiten Teil der Broschüre wird ein detaillierter Plan für die Durchführung der acht vorgesehenen Lektionen aufgestellt, wie dies für Deutschland iblich ist. Er ist theoretisch nach den oben angefürten Grobzielen strukturiert, wobei nach Möglichkeit noch zusätzliche Feinziele erarbeitet werden können. Für den Einstieg wird in der ersten Stunde ein Fragebogen von den Schülern über ihre Urlaubsgewohnheiten ausgefüllt und ausgewertet. Daneben können Kurzreferate von 2 - 3 Schülern über eigene Ferienaufenthalte eingeplant werden.

Es folgen die Lektionen über die geschichtliche Entwicklung des Tourismus, Gründe für die Erschliessung des Mittelmeerraums, Bedeutung des Fremdenverkehrs für ein Land wie Spanien, Auswirkungen am Beispiel Benidorm, und am Schluss Projektarbeiten in der "Integrierungsphase". Diese sollten das selbständige Arbeiten der Schüler fördern, es werden drei Vorschläge gemacht: 1. Werbung für Benidorm (mittels Collagen aus Werbeprospekten), 2. Bürgerinitiative in einem fiktiven Nachbarort von Benidorm und 3 . Rollenspiel in einem Reisebiiro. Eine andere Abschlussmöglichkeit wäre ein Test, der in der Unterrichtseinheit abgedruckt ist (System Multiple Choice).
BICHSEL Ulrich/KURZ Rudolf: Indien - Entwickungsland zwischen Tradition und Fortschritt. Studienbücher Geographie, 120 S. zahlr. Abb., Verlag Sauerländer Aarau, 1982, Fr. 15.80.

Es handelt sich um eine Neuauflage der bereits in der Serie Geographica Bernensia erschienenen Broschüre. Neu sind die sehr gut ausgewählten Farbfotos, die von den Autoren selbst aufgenommen wurde.

Positiv ist vor allem zu diesem Band zu bemerken, dass das Hauptgewicht auf das ländliche Indien - das Dorf - gelegt wurde, leben doch noch heute zwei Drittel der Einwohner im ländlichen Gebiet. Oft wird jedoch in der Tagespresse nur über indische Städte und ihre Probleme geschrieben, und es entsteht ein verzerrtes Bild der indischen Realität.

Die Autoren gehen z.B. detailliert, mit Flurskizzen illustriert, auf die Abhängigkeit Kasten - Landbesitz ein. Was mir allerdings fehlt, sind die zusätzlichen Abhängigkeiten Kaste Bodenqualität - Bewässerungsmöglichkeiten. Nicht alle Böden eignen sich gleich gut für künstliche Bewässerung, und die Grundwasserbrunnen der niedern Kasten sind oft wenig tief und fallen in der Vormonsunzeit trocken. Die Besitzer der noch wasserführenden Brunnen sind jedoch höheren Kasten zugehörig und verweigern die Wasserentnahme. So kormt es jährlich in zahlreichen Dörfern zu Unruhen. Auch das Festhalten an traditionellen Bebauungs- und Bewässerungsmethoder (ausschliesslich durch tierische oder menschliche Energie) scheint mir weniger Ausdruck von innovationsfeindlichem Verhalten $z u$ sein, als ein ökonomisches Problem: wie soll der Kleinbauer oder Pächter Geld für elektrischen Strom resp. Kerosin für den Betrieb einer Pumpe aufbringen ? (beides in Indien unverhältnismässig teuer).

Das Kapitel über die Bevölkerungsentwicklung begegnet dem immer noch verbreiteten $\mathrm{Kli}$ schee der steigenden Geburtenraten, die in Wirklichkeit seit den 30 er Jahren rückläufig sind. Gründe sind sicher die Senkung der Sterberate von 4,7 ( $\varnothing$ Dekade 1911/21) auf 1,5 \& (1971/81) und die noch immer gänzlich fehlende staatliche Kranken-, Invaliden- und Altersversicherung.

Die Kapitel über Iandflucht und indische Städte scheinen mir weniger geglückt. Warum gerade Chandigarh als Beispiel für indische Stadtplanung ? Nach meiner Erfahrung (mehrmonatiger Aufenthalt) ist gerade diese Stadt schlecht an indische Verhältnisse adaptiert, sowohl in klimatischer wie sozioökonomischer Hinsicht (Probleme der Leerflächen und grossen Distanzen, was allerdings von den Autoren als Kritik vermerkt wurde) .

Lobenswert ist jedoch die Zusammenstellung der 5-Jahrespläne: nach ihren jeweiligen Zielsetzungen; den Mitteln, die zur Erreichung der Ziele eingesetzt wurden, und den Erfolgs- resp. Misserfolgstendenzen.

Der Subkontinent Indien scheint mir etwas zu global abgehandelt, eine Beschränkung z.B. nur auf die nordindische Region wäre sinnvoll gewesen. 